\title{
Investigation of Cellular Mechanics by Atomic Force Microscopy
}

\author{
Fabian Heinemann*, Anna Kaufmann ***, Jens Schäpe**, Reimer Stick**, Manfred Radmacher* \\ *Institut of Biophysics, University Bremen, Germany \\ **Institut of Cellbiology, University Bremen, Germany
}

Atomic force microscopy (AFM) can be used to investigate biological samples under physiological conditions and hence allows following the dynamics of biological processes. In addition, the application of forces allows elucidating the mechanical properties of samples under investigation.

In particularly, very interesting is the investigation of the mechanics during cellular dynamics of cells, e.g. cell division or migration. In eukaryotic cells the mechanical properties are mainly caused by the cytoskeleton and thus mechanical data reflect the status of the actin network. Changes in architecture or composition of the cytoskeleton, or in the activity of actin binding proteins can be picked up by mechanical measurements.

By loading locally the sample information on the mechanical properties e.g. of life cells can be obtained. In eukaryotic cells the stiffness is mainly determined by the actin cytoskeleton, so the very soft plasma membrane can be neglected. Since the actin cytoskeleton determines the morphology of the cell, but is also involved in many cellular processes the mechanical state of the cell gives information on the activity of the cell. From a physical point of view, the stiffness of the actin cytoskeleton depends on the density of actin fibers, its architecture, its degree of cross-linking, and internal stress generated e.g. by myosin. All these contributions can be elucidated by mechanical experiments and by bio-chemical modification of the activity of these components. E.g. reducing the activity of myosin results in a softening of the cytoskeleton, as has been shown by drugs inhibiting myosin light chain kinase (Y-27632 or BDM). When looking at cellular activity local changes in stiffness can be monitored and related to the activity of the actin cytoskeleton. For instance in cell division, a stiffening of the cleavage furrow can be detected before the formation of the cleavage furrow is visible in topographic data.

In other cells like bacteria, or when investigating the cell nucleus, it turns out that the cell membrane itself is the determinant of the mechanical response. E.g. in cell nuclei we could determine the contribution of Lamins, which form a dense network on the inside of the nucleus, on the stiffness of the nucleus. By expressing mutant lamins in the nucleus of Xenopus oocytes we are able to investigate mechanical reasons of several laminopathies, which has been hypothesized.

The mechanical response of the sample can be used to derive the material properties, e.g. the Young's modulus, if an appropriate model for data analysis is chosen. The mechanical response of a three-dimensional gel like the actin cytoskeleton, is best described by the Hertz model, whereas the response of the bacterial cell wall, or the nuclear envelope of cell nuclei, is best described by the theory of thin shells. From an experimental point of view, the Hertz model results in a non-linear force-indentation relation, whereas the shell theory predicts a linear relation ship. 


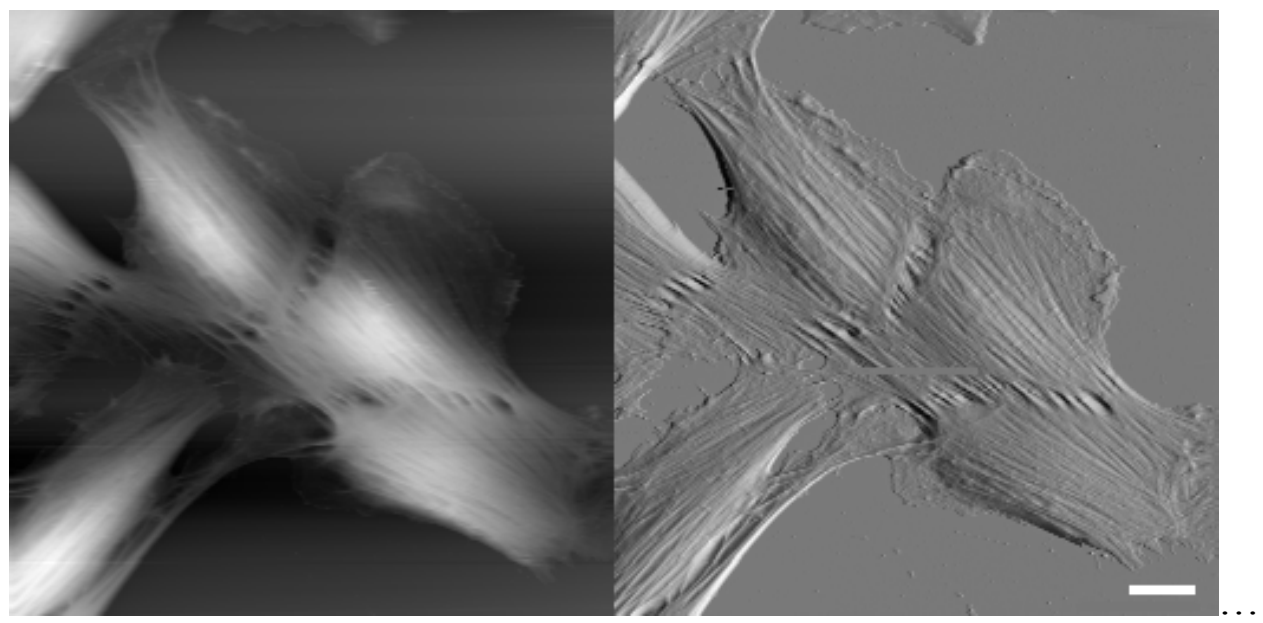

Contact mode AFM image of fibroblast cells. Scale Bar $5 \mu \mathrm{m}$.

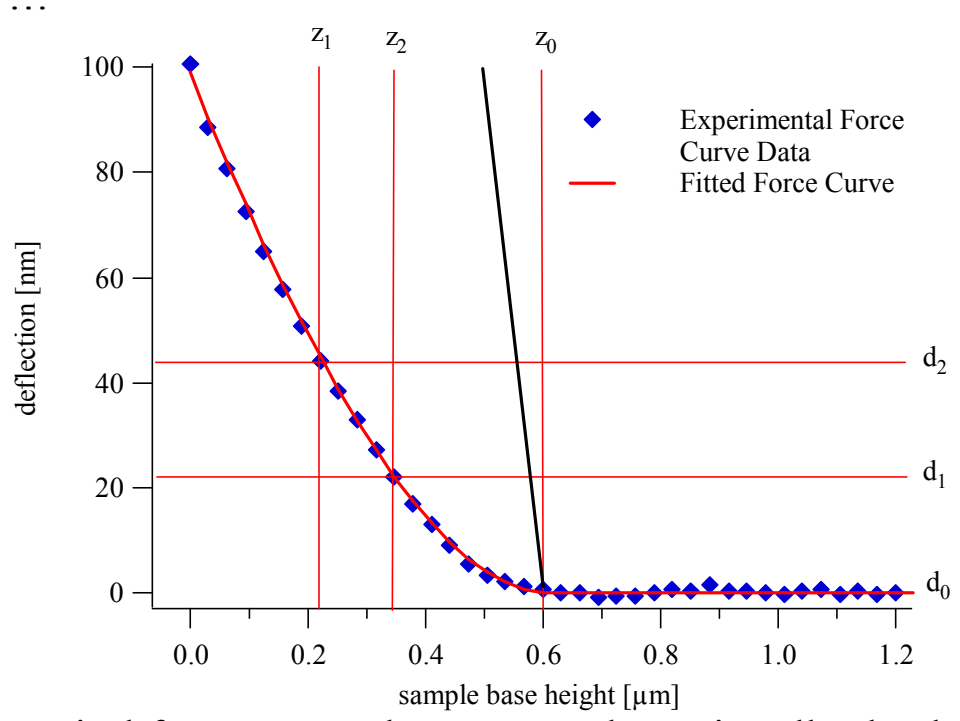

Typical force curve taken on an eukaryotic cell. The data are analyzed in a well-defined low force regime (depicted by the deflection values $\mathrm{d} 1$ and $\mathrm{d} 2$ ) and the Hertz model is fitted to the data. The quality of the fit is very good, since it matches the data very well even beyond the actual range of data, which have been used for the fit. 